\title{
LA PEDAGOGÍA HOSPITALARIA: CLAVE EN LA ATENCIÓN AL NIÑO ENFERMO Y HOSPITALIZADO Y SU DERECHO A LA EDUCACIÓN
}

\section{The key Hospital Pedagogy in the care of the sick and hospitalized child and their right to education}

\author{
M. a Isabel Calvo Álvarez \\ INICO-Facultad de Educación \\ Universidad de Salamanca \\ Correo-e: isabelc@usal.es \\ Recepción: I7 de noviembre de 2016 \\ Envío a informantes: is de enero de 2017 \\ Aceptación definitiva: 30 de marzo de 2017
}

Resumen: Los cambios a lo largo de la historia de la educación han favorecido la atención al niño enfermo. En las siguientes líneas nos acercamos a la Pedagogía Hospitalaria, sus orígenes, objetivos, presente y futuro. La Pedagogía Hospitalaria está traspasando la zona pediátrica para dar una respuesta integral al paciente mejorando su calidad de vida.

Palabras Clave: Pedagogía Hospitalaria; derecho a la educación; proyectos educativos; educador hospitalario.

Aвstract: Changes throughout the history of education have facilitated attention to the ill child. In the following lines, we approach Hospital Pedagogy, its origins, objectives, present and future. Hospital Pedagogy is crossing the pediatric zone to give an integral response to the patient, improving their quality of life.

KeY words: Hospital Pedagogy; education rights; educational projects; Teaching Hospital.

\section{Introducción}

\footnotetext{
7 scribir sobre Pedagogía Hospitalaria me devuelve a las aulas de la Facultad de Educación, en concreto a la asignatura de Pedagogía Terapéutica, impartida por la profesora Carmen Ortiz, donde por primera vez me acerqué no solo a este ámbito, sino también a la Educación Especial.
} 
Ortiz (1994) sitúa los orígenes de la Pedagogía Hospitalaria en el ámbito de la Educación Especial en los trabajos realizados por Itard, Decroly, Montessori..., quienes iniciaron la colaboración médico-pedagógica en hospitales, asilos y psiquiátricos. En este contexto, la Pedagogía Hospitalaria es la disciplina que da respuesta a las necesidades educativas, afectivas y sociales de las personas enfermas; planifica actividades que ayudan a la recuperación del paciente, y ofrece apoyo tanto a la persona como a su familia. Para Debesse y Mialaret (1986), citados por Lizasoáin y Polaino-Lorente (1996: 15) «es pedagogía en tanto que constituye el conjunto de aquellos medios puestos en acción para llevar a cabo la educación y es hospitalaria en tanto que se realiza y se lleva a cabo dentro del contexto hospitalario o en otros ámbitos relacionados con él». Cárdenas y López (2006: 59) consideran la Pedagogía Hospitalaria como una rama de la Pedagogía Social, «un intento de continuar con el proceso educativo de aquellas personas que están enfermas o convalecientes, de los familiares del enfermo $\mathrm{y}$ de todas aquellas personas que interfieren en la vida del paciente». La intervención que se lleva a cabo desde el ámbito de la Pedagogía Hospitalaria sitúa, cada vez más, al paciente en el centro de la misma, con el fin de mejorar su estado de salud y calidad de vida, hacer frente a su enfermedad, reducir sus necesidades formando parte de su proyecto educativo, proyecto que debe tener presente a la familia, al personal sanitario y/o cualquier agente de intervención en la atención al paciente; el niño enfermo adquiere los aprendizajes básicos de su edad: cualquier contenido que se trabaja en el aula parte de la situación de enfermedad; la finalidad es prevenir y evitar la marginación del proceso educativo de los niños hospitalizados, favorecer el desarrollo psicosocial, emocional e intelectual tanto en el hospital como en su domicilio (Polaino Lorente, 1990; Lizasoáin, 2000, 2003).

Al inicio, la Pedagogía Hospitalaria «... ha estudiado la realidad de la educación frente a la enfermedad, tomando en cuenta muchos de los complejos factores que supone este tipo de atención, proponiendo una pedagogía ajustada, que responda eficientemente a esta realidad» (Violant, Cardone, Monsalve y Márquez, 20I2: 3). En un primer momento se centró en el contexto escolar (aulas, programas, recursos educativos), hoy por hoy se ha extendido a todos los pacientes, personal sanitario y población que está en el hospital respondiendo a las necesidades reales aquí y ahora (asistenciales, educativas, psicológicas, etc.). De esta forma, maestros, pedagogos, psicólogos, educadores sociales... pretenden en el ámbito hospitalario, a través de un plan de atención individualizado, mejorar la calidad de vida durante la hospitalización y la enfermedad con el fin de conseguir la normalización en la vida del paciente y su familia y su adaptación a su vida cotidiana superada su estancia en el hospital y su enfermedad, principalmente en la etapa infantil (Cárdenas y López, 2006).

Blanco y Latorre (20II: 586) concretan en cuatro los objetivos de la Pedagogía Hospitalaria: el desarrollo de los aspectos cognitivos; el desarrollo motriz; el desarrollo afectivo y el desarrollo social. Hernández y Rabadán (2013: 179) especifican otro objetivo a nuestro entender clave en la atención al niño enfermo: «realizar los análisis pertinentes y la detección de necesidades educativas de aquellos pacientes que han sido hospitalizados con una previsión de estancias medias o largas» y Gutiez y Muñoz (2013: 133) definen objetivos específicos como:

- Continuar el currículum establecido con carácter general, en el nivel correspondiente y con las oportunas adaptaciones curriculares. 
- Facilitar la integración del niño en su nivel de escolarización al finalizar su hospitalización.

- Paliar el retraso escolar con las actividades escolares.

- Conseguir a través de las distintas áreas curriculares que sea capaz de valorar la dimensión real de su enfermedad, evitando así la angustia y el aislamiento.

- Procurar dar un carácter positivo y formativo a los tiempos libres y de ocio en el hospital (carácter educativo).

- Diseñar actividades de carácter formal e informal para mejorar los aspectos psicoafectivos.

- Estimular la participación de otras entidades o grupos para atender a los niños hospitalizados.

Así, se puede afirmar que se han ampliado los contextos, momentos y destinatarios sobre los que interviene la Pedagogía Hospitalaria: sacándola de la planta de pediatría y acercándola a los hospitales de día y a los domicilios, atiende no solo al paciente, sino también a su familia; y todo ello debido a los avances producidos en el ámbito de la salud, en la concepción de la educación y de la escuela, a la disminución del período de estancia en el hospital y a la detección de necesidades nuevas y diferentes a las identificadas hasta el momento.

Las necesidades, enfermedades, procesos y recursos cambian, y con ello también el cómo, dónde y cuándo planificar e implementar el plan de intervención. Los principios de inclusión, normalización e igualdad de oportunidades se han convertido en la base de la intervención en la persona enferma y/o hospitalizada, con el fin de conseguir una atención biopsicosocial que genere una mejora en la calidad de vida del paciente, minimizando las consecuencias de la enfermedad y/u hospitalización, para ello es imprescindible relacionar el ámbito de la salud y el ámbito educativo (Ortiz, 200I; Violant, Molina y Pastor, 20Ir; Hernández y Rabadán, 20I3). Cualquier avance y cambio no debe dejar a un lado las fases del proceso de ingreso en el hospital, punto de partida para poner en marcha metodologías y recursos que respondan a las

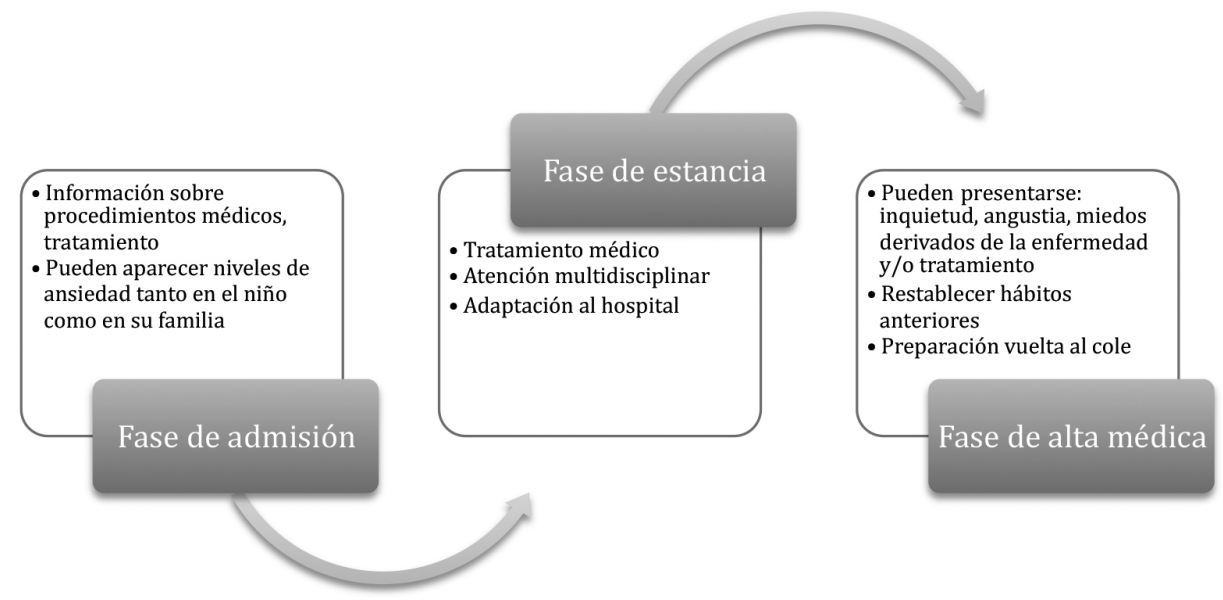

FIgURA I. Fases de estancia en el hospital. 
necesidades que surjan durante el proceso. La Figura I recoge las fases de estancia en el hospital siguiendo los trabajos de Ortiz (200I) González, Macías y García (2002) y Muñoz (20I3).

La atención al niño hospitalizado se asienta sobre la complejidad de las fases y las variables que intervienen en cada una de ellas. Para reducir la ansiedad, la angustia y otros problemas psicológicos que emergen durante la enfermedad y la hospitalización se debe planificar la hospitalización y elaborar proyectos de intervención global que cubran sus necesidades, ofreciendo diferentes apoyos como son el apoyo a la salud, el apoyo educativo, el psicológico y el social. El fin es conseguir que el desarrollo del niño se realice de la manera más normalizada posible, lo que supondrá diseñar desde proyectos de preparación a la hospitalización hasta proyectos de preparación para la vuelta a la colegio, es decir, establecer un plan de acción afín a las características del niño, la familia y el hospital, labor compartida por profesionales del ámbito de la salud, de la educación, la familia y los voluntarios.

\section{I. ... Un poco de historia}

Desde los orígenes de la Pedagogía Hospitalaria hasta hoy se ha dado respuesta a las necesidades que la enfermedad y la hospitalización han generado en el niño enfermo; recordar que la hospitalización es el período de permanencia en un contexto hospitalario, y en ese tiempo se llevan a cabo acciones centradas en la enfermedad (diagnóstico, tratamiento...) (Ortigosa y Méndez, 200o), acciones que se han ampliado a la educación.

Periats y Granados (2015: I88) establecen que «La educación del niño debe garantizarse en cualquier contexto en el que transcurra su vida, siendo necesario proporcionarle una enseñanza global y sistémica». Además, lo consideran un factor esencial en el desarrollo educativo, formativo y afectivo de una serie de niños que, debido a una enfermedad, pasan un tiempo más o menos prolongado de su vida en un hospital y, por lo tanto, lejos de su colegio y entorno familiar y escolar ( $\mathrm{p}$. 206). Por ello, Caballero (2007: 156) señala que el objetivo de la educación para cualquier niño es «brindarles igualdad de oportunidades y hacer que desarrollen todas sus capacidades y habilidades dentro de un clima de tolerancia y respeto a las diferencias».

En España las aulas hospitalarias son espacios creados, mayoritariamente, en las plantas de pediatría por el Ministerio de Educación y Cultura, que surgen para atender a los niños hospitalizados y así cumplir el derecho a recibir educación. Es un derecho de los niños enfermos $y / o$ hospitalizados seguir $y$ tener igualdad de oportunidades. En este sentido, la Constitución española de 1978, en su artículo 27, recoge: «Todos los españoles tienen el derecho a la educación». Las primeras aulas hospitalarias pertenecieron a la Orden Hospitalaria de San Juan de Dios en los años cincuenta; les siguen aulas en diferentes hospitales de Madrid, Valencia y Oviedo. En 1974, el Hospital de Parapléjicos de Toledo puso en marcha cinco aulas hospitalarias y una biblioteca. Para responder a los ingresos producidos, a partir de I98I, por el Síndrome del Aceite Tóxico se incrementan las aulas en los hospitales españoles (Prendes, Sánchez y Serrano, 20I2). Y en 1982 la Ley de Integración Social de los Minusválidos (LISMI) en su artículo 29 estableció que: 
Todos los hospitales, tanto infantiles como de rehabilitación, así como aquellos que tengan servicios pediátricos permanentes, sean de la Administración del Estado, de los Organismos Autónomos de ella dependientes, de la Seguridad Social, de las Comunidades Autónomas y de las Corporaciones Locales, así como los hospitales privados, que regularmente ocupen cuando menos la mitad de sus camas, con enfermos cuya estancia y atención sanitaria sean abonadas con cargo o recursos públicos, tendrá que contar con una sección pedagógica para prevenir y evitar la marginación del proceso educativo de los alumnos en edad escolar internados en dichos hospitales.

En 1983, se publica el Real Decreto II74/1983, de 27 de abril, sobre Educación Compensatoria que propugna el desarrollo de Programas para grupos en desventaja educativa con el fin de disminuir su discriminación; uno de los grupos que requieren una atención educativa derivada de unas características singulares como es el caso de la enfermedad son los niños enfermos y/o hospitalizados.

Hablar de alumnos con Necesidades Educativas Especiales (nee) derivadas de enfermedad nos traslada a 1978, en concreto a Inglaterra, donde un grupo de expertos se reunieron y elaboraron el documento por todos conocido «Informe Warnock», en el que se recoge por primera vez que las necesidades pueden cambiar tanto por las condiciones personales de la persona como por las condiciones del contexto, incorporando también la idea de que la persona puede necesitar en cualquier momento de su vida atención, intervención especial que le ayude a su inclusión. Introduce la filosofía de la integración frente al modelo segregado incorporando en el grupo de alumnos con nee a los niños enfermos ingresados en los centros hospitalarios. El informe hace referencia a cómo dar respuesta a las necesidades educativas; en aquel momento se centra en las adaptaciones curriculares, recursos materiales y espaciales y técnicas de enseñanzas especializadas, pidiendo una educación para los niños hospitalizados y un espacio adecuado para la educación. Es a partir de este informe cuando se inicia un cambio de paradigma en la atención a la diversidad. Hoy sabemos que una escuela inclusiva que da respuesta a la diversidad de su alumnado no requiere únicamente de adaptaciones curriculares y recursos especiales para unos pocos, sino trabaja por y para cada uno de sus alumnos identificando las barreras para el aprendizaje y la participación y buscando la presencia, la participación y el éxito de sus estudiantes logrando el aprendizaje y la participación de cada niño (Grau, 1993; Supinos, 1993; Ortiz, 20or; Blanco, 2008; Ainscow y Miles, 2008; Giné, 2009).

Esta idea queda recogida en el Real Decreto 334/1985, de 6 de marzo, de Ordenación de la Educación Especial, centrada en la dotación de aulas y profesores hospitalarios para atender las necesidades de los niños enfermos y las propias Administraciones Educativas pueden concertar servicios con instituciones sanitarias públicas, el fin es prevenir y evitar la marginación del proceso educativo de los niños en edad escolar en los centros hospitalarios.

En I990 se aprueba la Ley Orgánica I/1990, de 3 de octubre, de Ordenación General del Sistema Educativo (LOGSE). No recoge específicamente al alumnado enfermo, pero determina que los poderes públicos diseñarán acciones para compensar situaciones desfavorables, en este caso hablar de alumno enfermo y/o escolarizado es hablar de necesidades educativas especiales derivadas de condiciones como es el caso de la enfermedad y/o hospital. Un año después en 1991 se elabora el documento "Aulas Hospitalarias» y se crea el Programa de Educación Compensatoria para las mismas. 
El Real Decreto 696/1995, de 28 de abril, de Ordenación de la Educación de los Alumnos con Necesidades Educativas Especiales, y el Real Decreto 299/1996, de 28 de febrero, de Ordenación de las acciones dirigidas a la Compensación de Desigualdades en Educación, recogen la creación de escuelas en los hospitales y la atención al niño enfermo en su domicilio respectivamente de los alumnos de educación infantil, educación primaria y educación secundaria obligatoria, dando la opción al Ministerio de Educación y Ciencia de establecer convenios con asociaciones sin ánimo de lucro para implementar los programas de atención domiciliaria. La tecnología llegará a las aulas hospitalarias gracias al convenio firmado en 1998 entre los Ministerios de Educación y Cultura y de Sanidad y Consumo y el Instituto Nacional de Salud (INSALud).

La Circular del I2/II/I996 de la Dirección Nacional de Centros Educativos establece los criterios generales para la organización de las actuaciones dirigidas a la atención de los alumnos hospitalizados y convalecientes con los objetivos de proporcionar atención educativa a los niños hospitalizados, favorecer la continuidad del proceso aprendizaje, las relaciones socioafectivas y fomentar la utilización del tiempo libre y de ocio en el hospital.

La Ley Orgánica 2/2006, de 3 de mayo, de Educación, establece en su título II, capítulo II, que para hacer efectivo el principio de igualdad en el Derecho a la Educación las Administraciones Públicas «desarrollarán acciones de carácter compensatorio en relación con las personas, grupos y ámbitos territoriales que se encuentren en situaciones desfavorables y proveerán los recursos económicos y los apoyos precisos para ello». Y la Ley Orgánica 8/2013, de 9 de diciembre, para la Mejora de la Calidad Educativa (LOMCE), establece que el alumnado adquirirá competencias a lo largo de su escolarización que favorezcan su desarrollo personal, social y profesional y su inclusión en la sociedad y en el apartado de Equidad en la Educación, capítulo II, Compensación de las desigualdades en educación, recoge que las Administraciones Públicas proveerán los apoyos y los recursos económicos necesarios para dar respuesta a situaciones desfavorables. La atención integral al alumnado con necesidad específica de apoyo educativo se iniciará desde el mismo momento en que dicha necesidad sea identificada y se regirá por los principios de normalización e inclusión.

Finalmente, el Real Decreto Legislativo I/20I3, de 29 de noviembre, por el que se aprueba el texto refundido de la Ley General de derechos de las personas con discapacidad y de su inclusión social en su artículo 20 recoge:

Los hospitales infantiles, de rehabilitación y aquellos que tengan servicios pediátricos permanentes, ya sean de titularidad pública o privada que regularmente ocupen al menos la mitad de sus camas con pacientes cuya estancia y atención sanitaria sean financiadas con cargo a recursos públicos, deberán contar con una sección pedagógica para prevenir y evitar la marginación del proceso educativo de los alumnos de edad escolar ingresados en dichos hospitales.

Es importante recordar que las Comunidades Autónomas tienen competencia en materia educativa y sanitaria por lo que varía el funcionamiento y organización de las aulas hospitalarias, la atención domiciliaria, los recursos y su denominación. Atienden a alumnos de edades y niveles educativos diferentes y tienen como fin normalizar la estancia del niño en el hospital, la atención educativa, la mejora la calidad de vida del niño enfermo y/o hospitalizado, prevenir y evitar que los niños se sientan marginados debido a su enfermedad. Para Caballero (2007: 153) el aula hospitalaria debe ser 
M. ${ }^{\mathrm{a}}$ ISABEL CALVO ÁLVAREZ

contemplada «[...] como una vía para poder hablar de una educación inclusiva, ya que se estaría asumiendo el desafío de integrarlos satisfactoriamente a la sociedad desde la misma realidad de los niños y/o adolescentes internados en instituciones médicas». Las aulas, en general, cuentan con material educativo, escolar, artístico, bibliográfico, nuevas tecnologías y con juegos y juguetes.

Al mismo tiempo, en 1984 , la Nacional Association for the Welfare of Children in Hospital (NAWCH) de Reino Unido, siguiendo las recomendaciones del Gobierno de Reino Unido, centradas en la necesidad de poner en marcha actividades educativas y lúdicas para los niños hospitalizados garantizando el acompañamiento de sus padres durante su enfermedad, publicó una carta ( 23 derechos) sobre los derechos de los niños hospitalizados que en 1986 el Parlamento Europeo hizo suya y la transmitió al Consejo de Europa, a la UnICEF y la Organización Mundial de la Salud, convirtiéndose, así, en la Carta Europea de los Derechos del Niño Hospitalizado, base en la atención al niño enfermo. En el año 2000 la asociación HOPE (Hospital Organisation of Pedagogues in Europe) aprueba la Carta Europea sobre el Derecho a la Atención Educativa de los Niños y Adolescentes Enfermos. En 2009 la Asamblea General de REDLACEH (Red Latinoamericana y del Caribe por el Derecho a la Educación de Niños, Niñas y Jóvenes Hospitalizados y en Tratamiento) aprueba la Declaración de los Derechos del Niño, Niña o Joven Hospitalizado o en Tratamiento de América Latina y el Caribe en el ámbito de la Educación, validada por el Parlamento Latinoamericano el 22 de noviembre de 2013 .

\section{Experiencias previas}

Son muchos los trabajos que recogen las consecuencias derivadas de la enfermedad y las alteraciones de la hospitalización determinadas por variables como la edad, las experiencias previas, el diagnóstico, el desarrollo cognitivo, la hospitalización, la coordinación de servicios... (Figura 2) (Rodríguez Marín, 1986; Polaino-Lorente, I990; Ortigosa y Méndez, 2000; Quiles et al., 2003; Grau y Ortiz, 20or; Guillén y Mejía, 2002; Serradas et al., 2002; Lizasoáin, 2003; Fernández y López, 2006; Romero y Alonso, 2007; Muñoz, 2013; Serrano y Prendes, 2015). Para reducir estas consecuencias
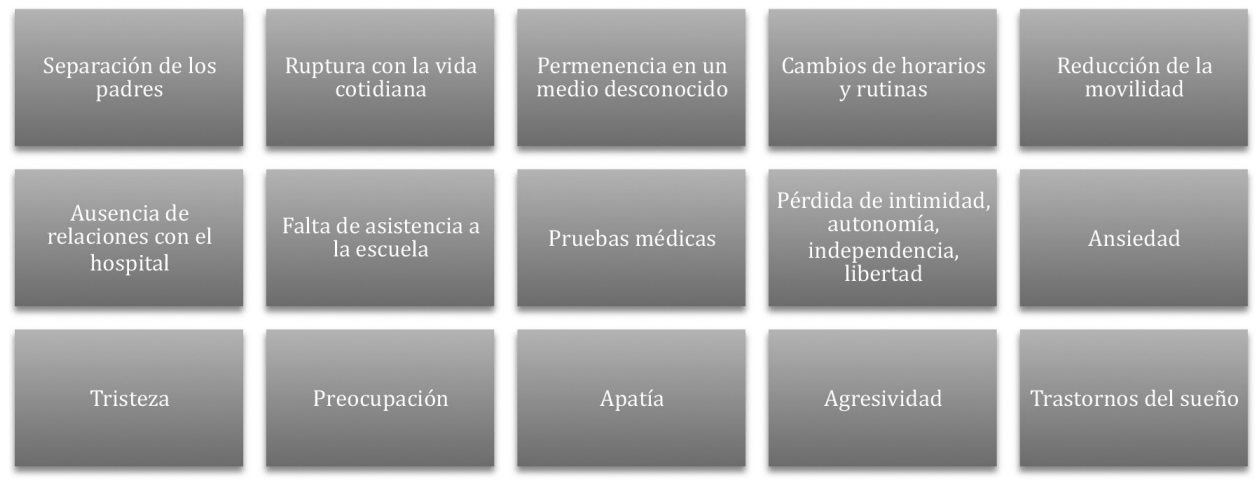

FIgURA 2. Consecuencias derivadas de la enfermedad y la hospitalización infantil. 
y el impacto diferencial que en el niño y su familia producen la enfermedad y la hospitalización es clave diseñar una intervención que englobe todas las dimensiones, que favorezca la adaptación y prevenga los efectos negativos del hospital y del tratamiento médico, que mejore su calidad de vida y contribuya a su felicidad (Calvo y Ortiz, 2003).

¿Quién da respuesta a las necesidades educativas? Los encargados de atender al niño en el hospital son los maestros hospitalarios, profesores de educación Infantil, Primaria y Secundaria; tienen formación y perfiles diferentes. En la actualidad en España no se cuenta en la formación inicial del Grado en Maestro con materias que preparen para trabajar en este ámbito. Destacar que la asignatura (optativa) de Pedagogía Hospitalaria y/o asignaturas afines se imparten en algunas Universidades como Burgos, Madrid, Navarra, Valencia y Santiago de Compostela (en algunas titulaciones han desaparecido como el caso de la optativa en la Diplomatura de Educación Social de la Facultad de Educación de la Universidad de Salamanca), acercando al alumnado al concepto de Pedagogía Hospitalaria, características del aula hospitalaria y necesidades del niño enfermo.

A día de hoy las aulas y el hospital son un lugar de «prácticas» para maestros, pedagogos y educadores sociales. La realidad señala la necesidad de incluir en los Grados de Educación contenidos centrados en el concepto de salud, la enfermedad, la muerte, los problemas psiquiátricos, el funcionamiento del sistema sanitario, la organización del hospital y de la planta de pediatría; créditos centrados en las funciones, estrategias de intervención y aptitudes de otros profesionales que intervienen en el proceso; competencias en las tecnologías de la información y la comunicación para favorecer el desarrollo y la educación del niño y del joven enfermo, y, finalmente, otro factor clave en la formación de todos los profesionales: conocer cómo informar, asesorar y acompañar a las familias.

El fin es la humanización de los hospitales y para ello se tiene que involucrar y formar no solo a los maestros, sino también a profesionales de la educación como pedagogos y educadores sociales, y a profesionales del ámbito de la enfermería y/o fisioterapia, en competencias emocional, creativa, comunicativa, colaborativa y en actitudes y valores en torno al trabajo en el hospital y a la diversidad. Es decir, se está hablando de una formación inicial y continua en constante cambio que forme equipos interdisciplinares que garanticen la calidad de vida del paciente y la calidad de los servicios.

Ante esta realidad ¿qué debe incluir un programa/proyecto hospitalario para lograr el derecho a una educación de calidad y mantener y/o mejorar su calidad de vida?

Cualquier actividad que se implemente en el hospital para alcanzar los objetivos a corto y medio plazo requiere la colaboración y coordinación de todos los agentes implicados en la atención al niño enfermo y hospitalizado, lo que supone crear y/o mantener equipos interdisciplinares que trabajen en el hospital, en la escuela, con la familia y en la comunidad (Ochoa, 2002; Castillo, 2005, Ullán, 2005).

Organizar y diseñar proyectos implica dar respuesta a las necesidades surgidas en el contexto hospitalario y/o derivadas de la enfermedad (salud, curriculares, psicopedagógicas, emocionales...); deben responder a la situación de cada paciente y centrarse en la dimensión educativa, la dimensión lúdica y la dimensión psicológica reduciendo la ansiedad, el estrés, los miedos; previniendo las alteraciones emocionales, el aburrimiento; aportando seguridad, confianza y colaborando en la adaptación del niño al hospital y a la enfermedad (Bermúdez y Torío, 2007; Bermúdez y Torío, 20I2; Linzasoáin, 20I5). 
La implementación de propuestas educativas conlleva utilizar variedad de recursos, técnicas y metodologías participativas e innovadoras que contribuyen a la socialización, la inclusión, la comunicación, la creatividad; fomentarán actitudes positivas, espacios de aprendizaje e intercambio de experiencias; el fin es reducir los efectos de la enfermedad y la hospitalización, no duplicar esfuerzos y recursos. Toda actividad se ofrecerá como alternativa a todos los niños sin distinción del número de días de estancia en el hospital.

La heterogeneidad de las personas, la duración de la hospitalización, los miedos, fobias, etc., son variables que favorecen el trabajo y el aprendizaje por proyectos, a través de los cuales tanto el paciente como los profesionales se convierten en los protagonistas de la intervención y se favorece la participación de todos en el proceso partiendo de su historia personal, proceso clínico y estado de ánimo. Se llevarán a cabo en el aula hospitalaria, en los hospitales de día, con pacientes en UCI, en servicios específicos, con los acompañantes del enfermo potenciando habilidades, conocimientos y actitudes positivas hacia la enfermedad, la hospitalización, los agentes de intervención, etc.

A continuación se recogen diferentes proyectos, experiencias, recursos y actividades que se han puesto en marcha en el contexto hospitalario. Algunos han finalizado, otros continúan con cambios importantes y otros han dado lugar a nuevos proyectos; sin embargo, todos demuestran los beneficios de la Pedagogía Hospitalaria tanto en el hospital, en el ámbito domiciliario como en el contexto escolar, en base a los pilares de la educación del siglo XXI «Aprender a conocer, aprender a ser, aprender a aprender y aprender a convivir». Proyectos multidisciplinares a través de convenios entre la Consejería de Sanidad, asociaciones, fundaciones, hospitales... que ayudan a mejorar la estancia y la calidad de vida de los pacientes en el hospital, a romper la rutina hospitalaria. Proyectos que apoyan a profesores de centros educativos, que ayudan al niño a contar su experiencia, expresar sentimientos, emociones, actividades que ayudan a relacionarse a las familias, programas de sensibilización y divulgación y mucho más.

Tabla i. Proyectos implementados en el ámbito hospitalario.

\begin{tabular}{|l|l|}
\hline \multicolumn{1}{|c|}{ Proyecto } & \multicolumn{1}{c|}{ Descripción } \\
\hline AIT EILE & $\begin{array}{l}\text { Objetivo: Favorecer la comunicación entre los niños in- } \\
\text { gresados minimizando los efectos negativos de la hospi- } \\
\text { talización. } \\
\text { Contexto: Hospitales de Irlanda. }\end{array}$ \\
\hline COMENIUS & $\begin{array}{l}\text { Objetivo: Intercambiar experiencias entre países para niños } \\
\text { de la etapa de educación obligatoria. } \\
\text { Contexto: Hospitales europeos. }\end{array}$ \\
\hline PROYECTO KLINI & $\begin{array}{l}\text { Objetivo: Abrirse a Europa a través de las nuevas tecno- } \\
\text { logías a través de una revista escolar realizada por jóvenes } \\
\text { hospitalizados. } \\
\text { Contexto: Luxemburgo, Francia, Bélgica, Italia y Alemania. }\end{array}$ \\
\hline $\begin{array}{l}\text { Proyecto Europeo Paintfolio, } \\
\text { dentro del Programa Eras- } \\
\text { mus+2OI4 }\end{array}$ & $\begin{array}{l}\text { Objetivo: Compartir las aulas hospitalarias, a través de una } \\
\text { página web, los valores culturales de los distintos países me- } \\
\text { diante la pintura. } \\
\text { Contexto: Hospitales europeos, coordinado por el aula hos- } \\
\text { pitalaria de Móstoles (Madrid). }\end{array}$ \\
\hline
\end{tabular}




\begin{tabular}{|c|c|}
\hline Proyecto & Descripción \\
\hline Proyecto lectura en el hospital & $\begin{array}{l}\text { Objetivo: Promocionar de lectura como una herramienta } \\
\text { terapéutica y de estimulación para el desarrollo integral de } \\
\text { los niños hospitalizados. } \\
\text { Contexto: Mundial. }\end{array}$ \\
\hline Ciberaulas Hospitalarias & $\begin{array}{l}\text { Objetivo: Crear espacios lúdico-educativos de entreteni- } \\
\text { miento, comunicación y aprendizaje. } \\
\text { Contexto: España. }\end{array}$ \\
\hline Proyecto ALTER & $\begin{array}{l}\text { Objetivo: Mejorar la atención educativa de los pacientes y } \\
\text { facilitar la formación del profesorado sobre las tic en las } \\
\text { aulas hospitalarias. } \\
\text { Contexto: Región de Murcia (España). }\end{array}$ \\
\hline Proyecto Mundo de Estrellas & $\begin{array}{l}\text { Objetivo: Mejorar la calidad de vida de niños y adolescentes } \\
\text { a través de la red en la que puedan interactuar y relacionarse } \\
\text { todos los niños y adolescentes ingresados en los hospitales } \\
\text { del Sistema Sanitario Público de Andalucía. } \\
\text { Proyecto lúdico-pedagógico que cuenta con variedad de } \\
\text { recursos adaptados a las distintas edades y desarrollos } \\
\text { madurativos. } \\
\text { Contexto: Comunidad Andaluza (España). }\end{array}$ \\
\hline Proyecto CurArte & $\begin{array}{l}\text { Objetivo: Desarrollar el juego, la educación artística y la } \\
\text { creatividad como recursos de salud y bienestar para niños y } \\
\text { adolescentes hospitalizados. } \\
\text { Ha ampliado su campo de acción con propuestas para adul- } \\
\text { tos y tercera edad. } \\
\text { Contexto: España. }\end{array}$ \\
\hline Proyecto House & $\begin{array}{l}\text { Objetivo: Cuando los niños crean un hospital a su medida. } \\
\text { Contexto: Madrid. }\end{array}$ \\
\hline $\begin{array}{l}\text { Fundación Ronald McDonald } \\
\text { House Charities }\end{array}$ & $\begin{array}{l}\text { Objetivo: Crear y mantener las Casas Ronald McDonald, } \\
\text { un «hogar fuera del hogar» para aquellos niños y sus fami- } \\
\text { lias que se trasladan fuera de su residencia habitual para re- } \\
\text { cibir tratamiento médico de larga duración. } \\
\text { Contexto: Mundial. }\end{array}$ \\
\hline Fundación Pequeño Deseo & $\begin{array}{l}\text { Objetivo: Conseguir que los sueños de los niños con en- } \\
\text { fermedades crónicas o de mal pronóstico se hagan realidad. } \\
\text { Contexto: España. }\end{array}$ \\
\hline Fundación Theodora & $\begin{array}{l}\text { Objetivo: Hacer más llevadera la estancia en el hospital de } \\
\text { niños y familiares que sea lo más humana posible. } \\
\text { Contexto: España. }\end{array}$ \\
\hline Fundación Aladina & $\begin{array}{l}\text { Objetivo: Ayudar a niños y adolescentes con cáncer y a sus } \\
\text { familias a través de diferentes acciones. } \\
\text { Contexto: España. }\end{array}$ \\
\hline Fundación Juegaterapia & $\begin{array}{l}\text { Objetivo: a través de proyectos como «El jardín de mi Hos- } \\
\text { pi» y «Estaciones lunares» mejorar la estancia en el hospital. } \\
\text { Contexto: España. }\end{array}$ \\
\hline
\end{tabular}


LA PEDAGOGÍA HOSPITALARIA: CLAVE EN LA ATENCIÓN AL NIÑO ENFERMO

Y HOSPITALIZADO Y SU DERECHO A LA EDUCACIÓN

M. ${ }^{\mathrm{a}}$ ISABEL CALVO ÁLVAREZ

\begin{tabular}{|l|l|}
\hline \multicolumn{1}{|c|}{ Proyecto } & \multicolumn{1}{c|}{ Descripción } \\
\hline Proyecto Sentido del humor & $\begin{array}{l}\text { Objetivo: Mejorar el bienestar del paciente. El humor para } \\
\text { evitar el estrés hospitalario, canalizar emociones, liberar ten- } \\
\text { siones, necesidad de sentirse protegido, seguro y tranquilo. } \\
\text { Contexto: España. }\end{array}$ \\
\hline RECURSOS-TIC & $\begin{array}{l}\text { Ayudan a reducir la sensación de aislamiento con respecto } \\
\text { al entorno habitual favoreciendo la creatividad, la imagina- } \\
\text { ción, motivación, etc. }\end{array}$ \\
\hline Revistas Hospitalarias & La Pecera, La Piruleta... \\
\hline $\begin{array}{l}\text { Trabajo a través de los cuentos, } \\
\text { los libros y el cine }\end{array}$ & $\begin{array}{l}\text { Ayudan a afrontar algunos de los problemas derivados de la } \\
\text { enfermedad y la hospitalización. }\end{array}$ \\
\hline Talleres & $\begin{array}{l}\text { Teatro, música, arte, ciencia, animación a la lectura en el aula } \\
\text { y en el hospital. }\end{array}$ \\
\hline Actividades artísticas & $\begin{array}{l}\text { El objetivo es contribuir a mejorar la experiencia de hospi- } \\
\text { talización ofreciendo la posibilidad de ocupar el tiempo de } \\
\text { manera creativa y normalizadora. }\end{array}$ \\
\hline Juegos & $\begin{array}{l}\text { Educativos, videojuegos, fomentan la actividad, ocupan su } \\
\text { tiempo libre, etc. }\end{array}$ \\
\hline
\end{tabular}

El fin de la intervención es reducir el impacto de la hospitalización en el menor y en sus familias, lo que significa disponer de un entorno apropiado para su edad, el derecho a la intimidad, el derecho a acceder a variedad de actividades que cubran sus necesidades y el derecho a la educación.

\section{3. ... Para terminar}

Se puede afirmar que no hay un único modo de acercarse a la enfermedad y a la hospitalización, del mismo modo que no hay un único plan o programa para implementar en el hospital y responder a las necesidades del paciente, cada acción puesta en marcha requerirá su implicación, él junto a los profesionales crearán su proyecto educativo. Este proyecto individual y personalizado no es incompatible con el diseño y desarrollo de programas y proyectos que involucren y comprometan a todos a trabajar por y para la atención al paciente y la mejora de los servicios sanitarios y educativos. Los niños enfermos son niños, lo que significa que quieren jugar, soñar, reír, llorar... quieren estar con sus amigos, sus juguetes, disponer de su espacio, ser escuchados, tomar decisiones, sentir que forman parte del grupo.

En todos los hospitales se están haciendo esfuerzos por humanizar los cuidados del niño hospitalizado, hacer más llevadera su estancia promoviendo oportunidades de aprendizaje y crecimiento personal sin olvidar que necesitan sentirse importantes y felices, y que el humor es clave en el proceso de recuperación y en la educación. En este momento las actividades puestas en marcha en la aulas hospitalarias, principalmente, se orientan a continuar con el proceso de aprendizaje de contenidos curriculares; fuera de la jornada escolar se diseñan actividades y proyectos lúdico-pedagógicos que desarrollan competencias que mejoran su desarrollo personal, favorecen su inclusión y hacen más agradable la estancia en el hospital. 
En estas últimas décadas se han dado pasos importantes en el ámbito de la Pedagogía Hospitalaria, se han creado nuevos servicios que responden a nuevas patologías..., pero no es suficiente; a corto plazo el objetivo es mejorar la atención al niño enfermo, su familia y el contexto y a medio-largo plazo ampliar esta atención a todos los pacientes, continuar trabajando para que, desde la política educativa, sanitaria y social, la práctica y la investigación proporcionen programas y recursos que contribuyan a una educación de calidad, a avanzar en la mejora de las condiciones de calidad de vida de la persona enferma y hospitalizada promoviendo la coordinación entre los agentes de intervención sin olvidar que lo primero es la salud.

El reto está en cómo preparar a los profesionales para trabajar en este entorno, para ello es necesario identificar sus necesidades formativas, conocer las variables que influyen en el proceso de intervención al paciente, generar investigaciones que permitan mejorar la educación en el hospital, conseguir hospitales y recursos seguros e inclusivos, mejorar las infraestructuras, aumentar los recursos humanos, eliminar y/o reducir barreras físicas, sociales y culturales, generar más y nuevos proyectos (intervención con jóvenes con problemas psiquiátricos, trabajar realidades como la muerte) y estar preparados para una rápida respuesta al paciente.

Pongamos en este reto todos los sentidos, la vida transcurre entre momentos dulces y amargos. ¿'A qué «huele», «suena» y «sabe» el hospital? Estemos atentos a lo que el paciente, la familia y los profesionales demandan para todos juntos lograr el fin de la Pedagogía Hospitalaria.

\section{Bibliografía}

Ainscow, M. (20ir) Respondiendo al desafío de la equidad en los sistemas educativos. Aula. Revista de Pedagogía de la Universidad de Salamanca, 17, 73-87.

Ainscow, M. y Miles, S. (2008) Por una educación para todos que sea inclusiva: ¿Hacia dónde vamos ahora? Perspectivas, XXXviII (I), I7-44.

Ayuntamiento de Móstoles (2015) Educación, Cultura y Promoción: Presentado un Proyecto Educativo Internacional coordinado por el Aula Hospitalaria de Móstoles. Recuperado el 8 de septiembre de 2016, de http://www.mostoles.es/es/noticias/educacion-cultura-promocion-cultura-presentado-proyecto-edu.

Bermúdez, M. T. y Torío, S. (2007) El voluntariado y la animación hospitalaria. En M. Cid y A. Peres (coords.) Educación Social, Animación Sociocultural y Desarrollo Comunitario (pp. 588-6or). Universidad de Vigo, Universidad de Tras os Montes e Alto Douro y Sociedad Iberoamericana de Pedagogía Social.

Bermúdez, M. T. y Torío, S. (2OI2) La percepción de las familias ante la animación hospitalaria: Estudio realizado en el hospital Materno-Infantil de Oviedo. Pedagogía Social. Revista Interuniversitaria, julio-diciembre, 20, 223-242.

Blanco, R. (2008) Construyendo las bases de la inclusión y la calidad de la educación en la primera infancia. Revista de Educación, 347, 33-54.

Blanco, F. J. y Latorre, M. ${ }^{a}$ J. (2OII) Implicaciones de la acción educativa en espacios específicos de proyección profesional en España. Educação e Pesquisa, São Paulo, 37, 3, 583-598.

Caballero, S. A. M. (2007) El aula hospitalaria en un camino a la educación inclusiva. Investigación Educativa, II (19), I53-16I.

Calvo, M. ${ }^{a}$ I. y Ortiz, M. ${ }^{a}$ C. (2013) Estudio de las necesidades percibidas por profesionales, padres y voluntarios en niños hospitalizados en el distrito universitario de Salamanca. En M. Á. Verdugo y F. B. Jordán de URRíEs (coords.) Investigación, innovación y cambio. 
LA PEDAGOGÍA HOSPITALARIA: CLAVE EN LA ATENCIÓN AL NIÑO ENFERMO

Y HOSPITALIZADO Y SU DERECHO A LA EDUCACIÓN

M. ${ }^{\mathrm{a}}$ ISABEL CALVO ÁLVAREZ

$V$ Jornadas Cientificas de Investigación sobre personas con discapacidad (pp. 689-70I). Salamanca: Amarú.

CÁrdenas, R. y López Noguero, F. (2006) Hacia la construcción de un modelo social de la Pedagogía Hospitalaria. Revista Interuniversitaria, I2-I3, 59-70.

Castillo, M. ${ }^{a}$ D. (2005) La coordinación en un aula hospitalaria. Revista de Aulas Hospitalarias, Castilla y León, 2, $\mathrm{I} 6$.

Circular del I2/II/1996 de la Dirección Nacional de Centros Educativos.

Constitución Española. Boletín Oficial del Estado, 29 de diciembre de 1978.

Fernández Hawrylak, M. (2000) La Pedagogía hospitalaria y el pedagogo hospitalario. Tabanque, I5, I39-I5O.

Fernández-Castillo, A. y López-Naranjo, I. (2006) Transmisión de emociones, miedo y estrés infantil por hospitalización. International Journal of clinical and Health Psychology, $6(3), 63 \mathrm{I}-645$.

Giné, Cl. (coord.) (2009) La educación inclusiva. De la exclusión a la plena participación de todo el alumnado. Barcelona: Horsori.

González, F.; Macías, E. y García, F. (2002) La Pedagogía Hospitalaria: reconsideración desde la actividad educativa. Revista Complutense de Educación, I3 (I), 303-365.

GraU, C. (1993) La integración escolar del niño con neoplasias. Barcelona: CEAC.

Grau, C. y Ortiz, C. (200I) La pedagogía hospitalaria en el marco de una educación inclusiva. Málaga: Ediciones Aljibe, s.A.

Guillén, M. y Mejía, A. (2002) Actuaciones educativas en aulas hospitalarias. Atención escolar a niños enfermos. Madrid: Narcea.

Gutiez, P. y MuÑoz, V. (20I3) Estrategias de intervención didáctica en el contexto de Aulas Hospitalarias. En C. SÁnchez Romero (coord.) Aplicación de estrategias didácticas en contextos desfavorecidos (pp. I25-I54). Madrid: UNED.

Hernández, E. y Rabadán, J. A. (2013) La hospitalización: un paréntesis en la vida del niño. Atención educativa en población infantil hospitalizada. Perspectiva Educacional, 52 (I), I67-I8I.

Hernández, E. y Rabadán, J. A. (20I4) Érase una vez... un cuento curativo. Atención educativa en población infantil hospitalizada a través de la literatura. Educatio Siglo XXI, 32 (2), I29-I5O.

Hospital Organisation of Pedagogues in Europe-hope (2000) Carta Europea sobre el Derecho a la Atención Educativa de los Niños y Adolescentes Enfermos. Recuperado el io de julio de 2016, de http:// www.hospitalteachers.eu/who/hope-charter/ hope-charter_sp.

Latinoamérica y el Caribe. Declaración de los derechos del niño, la niña o joven hospitalizado o en tratamiento de Latinoamérica y el Caribe en el ámbito de la educación. Red Latinoamericana y del Caribe Por el Derecho a la Educación de Niños y Jóvenes Hospitalizados o en Tratamiento (REDlaceH), 9 de septiembre de 2009.

Ley I3/1982, de 7 de abril, de Integración Social de los Minusválidos. Boletín Oficial del Estado, 30 de abril de 1982 (LISMI).

Ley Orgánica I/ı990, de 3 de octubre, de Ordenación General del Sistema Educativo (LOGSE).

Ley Orgánica 2/2006, de 3 de mayo, de Educación. Boletín Oficial del Estado, 4 de mayo de 2006 , 106.

Ley Orgánica 2/2006, de 3 de mayo, de Educación. Boletín Oficial del Estado, 4 de mayo de 2006 (LOE).

Ley Orgánica 8/2013, de 9 de diciembre, para la mejora de la calidad educativa. Boletín Oficial del Estado, io de diciembre de 2013 (LOMCE).

Lizasó́in, O. (2000) Educando al niño enfermo. Perspectivas de la Pedagogía Hospitalaria. Pamplona: Eunate.

Lizasó́in, O. (2003) Los retos de la atención educativa: del alumnado hospitalizado o convaleciente en el siglo xxi. En AA. vv. Memoria de las IX Jornadas de Pedagogía Hospitalaria (pp. II-I7). Guadalajara: Federación Española de Niños con Cáncer. 
Lizasoárn, O. (2015) Pedagogía Hospitalaria: Un contexto "fuera de contexto». I Congreso Internación de Pedagogía Hospitalaria en Colombia, I9 y 20 de febrero, 2014.

Lisasoáin, O.; Ochoa, B. y Sobrino, A. (2000) Parámetros comunes en la pedagogía hospitalaria europea. En Actas del IV Congreso Europeo de maestros y pedagogos en el hospital. Barcelona: PAU Educación, I63-I7I.

Lizasoáin, O. y Polaino-Lorente, A. (1996) La Pedagogía Hospitalaria como un concepto unívoco e innovador. Revista Comunidad Educativa. Madrid, 23I (3), I4-I6.

Lizasoáin, O. (2007) Hacia un modo conjunto de entender la Pedagogía Hospitalaria. Primera Jornada Nacional de Pedagogía Hospitalaria en Venezuela. La educación prioridad de vida, I-I5.

Muñoz Garrido, V. V. (20I3) Pedagogía Hospitalaria y Resiliencia. Tesis doctoral. Universidad Complutense de Madrid. Madrid.

OchoA, B. (2002) Reflexiones en torno al voluntariado hospitalario en Navarra. Pedagogía Social. Revista Interuniversitaria, 9, Segunda Época, 4I-52.

Ortigosa, J. M. y Méndez, F. X. (2000) Hospitalización infantil: repercusiones psicológicas. Madrid: Biblioteca Nueva.

Ortiz, M. a C. (1994) Pedagogía Hospitalaria. Revista Siglo Cero, 25 (5), 4I-45.

Ortiz, M. ${ }^{a}$ C. (20oI) Perspectivas de la pedagogía hospitalaria. En C. Grau y M. ${ }^{a}$ C. Ortiz La pedagogía hospitalaria en el marco de la educación inclusiva (pp. 19-55). Málaga: Aljibe.

Parlamento Europeo (1986) Carta Europea de los Niños Hospitalizados. Recuperado el 3 de junio de 20I6, de http://www.pediatriasocial.com/Documentos/cartaeuropea.pdf.

Peirats, J. y Granados, J. (2015) Las Unidades Pedagógicas Hospitalarias y el aprendizaje por Proyectos de Trabajo. Aula de Encuentro, I (17), I87-2II.

Polaino-Lorente, A. (1990) La Pedagogía Hospitalaria desde la perspectiva médica. En J. L. González-Simancas y A. Polaino-Lorente Pedagogía Hospitalaria. Actividades educativas en ambientes clínicos (pp. 27-79). Madrid: Narcea.

Prendes, M. P.; Sánchez-Vera, M. M. y Serrano, J. L. (20i2) Posibilidades educativas de las TIC en las aulas hospitalarias. Journal for Educators, Teachers and Trainers, 3 (I), 37-48.

Quiles, M. J.; Ortigosa, J. M. y Méndez, F. X. (2003) Preparación a la hospitalización y la cirugía. En J. M. Ortigosa-Quiles, M. J. Quiles-Sebastián y F. X. Méndez-Carrillo (coords.) Manual de psicología de la salud con niños, adolescentes y familia. Madrid: Pirámide.

Real Decreto II74/1983, de 27 de abril, sobre Educación Compensatoria.

Real Decreto 334/1985, de 6 de marzo, de Ordenación de la Educación Especial.

Real Decreto 696/1995, de 28 de abril, de Ordenación de la Educación de los Alumnos con Necesidades Educativas Especiales.

Real Decreto de 299/1996, de 28 de febrero, de Ordenación de las acciones dirigidas a la Compensación de Desigualdades en Educación.

Real Decreto Legislativo I/2013, de 29 de noviembre, por el que se aprueba el Texto Refundido de la Ley General de derechos de las personas con discapacidad y de su inclusión social. Boletin Oficial del Estado, 289, de 3 de diciembre de 2013.

Romero, K. y Alonso, L. (2007) Un modelo de práctica pedagógica para las aulas hospitalarias: el caso del Hospital Universitario de Los Andes. Revista de Pedagogía, 28 (83), septiembre-diciembre, 407-44I.

Rosselló, M.; Iglesia, B. de la; Paz-Lourido, B. y Verger, S. (2015) Necesidades de formación psicopedagógica para la atención de niños con enfermedad crónica: percepciones de enfermería hospitalaria. Revista da Escola de Enfermage USP, 49 (I), 37-43.

SÁnCHEz, A. y López, D. (2016) Evaluación de la respuesta educativa al alumnado de aulas hospitalarias en la provincia de Almería. Revista Española de Discapacidad, 4 (I), 83-96.

Segura, M.; Candioti, C. y Medina, C. J. (2007) Las Tic en la Educación: panorama internacional y situación española. Documentación presentada en XXII Semana Monográfica de la Educación: Las TIC en la Educación: retos y posibilidades, Madrid, España. Recuperado el I de septiembre de 2016, de http://www.oei.es/tic/DocumentoBasico.pdf. 
Serradas, M.; Ortiz, M. ${ }^{a}$ C. y De Manueles, J. (2002) Necesidad de asistencia educativa al niño hospitalizado. Enseñanza, 20, 243-258.

Serrano, J. L. y Prendes, M. P. (20I5) Integración de las Tic en aulas hospitalarias como recursos para la mejora de los procesos educativos. Estudios sobre Educación, 28, I87-210.

Ullán, A. M. (2005) Proyecto curarte de juego y creatividad en niños hospitalizados. Revista de Aulas Hospitalarias. Castilla y León, 2, 4-5.

Violant, V.; Cardone, P.; Monsalve, C. y Márquez, L. (2OI2) Multidimensionalidad de la pedagogía hospitalaria desde la transdisciplinariedad. Barcelona: CIAD, Universitat de Barcelona.

Violant, V.; Molina, M. C. y Pastor, C. (2009) Pedagogía Hospitalaria: Necesidades, ámbitos y metodología de intervención. Santiago de Chile: Ministerio de Educación, Gobierno de Chile.

Violant, V.; Molina, M. C. y Pastor, C. (20II) Pedagogía Hospitalaria. Bases para la atención integral. Barcelona: Laertes. 